\title{
Analytical Model Development for Unified 2D Electron Gas Sheet Charge Density of AlInN/GaN MOSHEMT
}

\author{
Gaini Amarnath, Trupti Ranjan Lenka
}

\begin{abstract}
We have developed a unified analytical model for computation of $2 \mathrm{D}$ electron gas sheet charge density in AlInN/GaN metal-oxide-semiconductor high electron mobility transistor device structure. This model has been developed by incorporating the variation in lowest three energy sub-bands and Fermi level energy in the quantumwell with respect to gate voltage. We noticed that the dependency of lowest sub-band energy with Fermi energy having two fields, which are the lowest sub-band energy is greater and lesser than the Fermi level energy. According to these two fields, we have developed the fermi energy and sheet charge density expressions in each field. By combining each field of the models, developed a unified 2D electron gas sheet charge density model. The Fermi level and sheet charge density are interdependent in the model development. The developed model results are compared with TCAD simulation results and obtain a good consistency between them. This model is fitted to other metal-oxidesemiconductor high electron mobility transistor devices also with modifications in related physical values.
\end{abstract}

\section{Keywords-2DEG, AlInN/GaN, MOSHEMT, TCAD}

\section{INTRODUCTION}

$\mathbf{F}$ ROM the past few years, AlInN/GaN high electron mobility transistors (HEMTs) are representing an attractive performance in high frequency, low noise, high switching voltage and high power areas [1-3]. These devices have been studied systematically as a replacement to $\mathrm{AlGaN} / \mathrm{GaN}$ HEMTs. The primary benefit of AlInN/GaN HEMTs device is that use of thin AlInN barrier and it is nearly lattice matched to $\mathrm{GaN}$. It provides a high $2 \mathrm{D}$ electron gas (2DEG) sheet charge density $\left(\mathrm{n}_{\mathrm{s}}\right)$ in the channel due to large spontaneous polarization charges present and also minimized short channel effects. However, these devices are affected by gate leakage current due to thin barrier material and restricted Schottky barrier thickness resulting in low off-state behavior and poor forward gate swing. In this situation, metal-oxidesemiconductor HEMTs (MOSHEMTs) are introduced to overcome the mentioned problems [3]. The GaN-based MOSHEMT devices are using the gate dielectric/oxide material as $\mathrm{SiN}$ [4], $\mathrm{SiO}_{2}$ [5], $\mathrm{Al}_{2} \mathrm{O}_{3}$ [6], $\mathrm{HfO}_{2}$ [7], $\mathrm{La}_{2} \mathrm{O}_{3}$ [8], $\mathrm{AlN}$ [9], $\mathrm{LaLuO}_{3}$ [10], $\mathrm{MgCaO}$ [3]. Among these oxides, $\mathrm{HfO}_{2}$ is considered in this work.

The 2D electron gas sheet charge density is formed in the quantum well of hetero-interface and it is the key principle to the further heterostructures device study. The 2DEG sheet charge density modeling is a primary requirement in the efficient DC model development for these devices. So, the authors have shown interest in developing physics related unified analytical model for ns. The development of ns has to be calculated from the prominent charge control expression [11] as a selfconsistent Schrodinger's and Poisson's equations in the triangular potential well.

In this work, we develop the fermi energy and 2D electron sheet charge density expressions in two different fields and combining to develop a unified 2D electron gas sheet charge density model. In model development, the Fermi-Dirac distribution function is multiply with a density-of-states function associated with the quantumwell electric field. Also, investigate the effects on sub band energy levels and ns with respect to varying the oxide thickness which is distinctive feature of this work.

\section{2D ELECTRON GAS SHEET CHARGE DENSITY MODEL}

To develop the model, we have considered $\mathrm{HfO}_{2} / \mathrm{AlInN} / \mathrm{GaN}$ MOSHEMT device structure as shown in figure 1 . The symbols of the definition and parameters used in this work are listed in table 1. In hetero interface structure, such as $\mathrm{HfO}_{2} / \mathrm{AlInN} / \mathrm{GaN}$ MOSHEMT the sheet charge density is created at the interface and accumulated in the quantum potential well. This charge density is the 2DEG sheet charge density $\left(n_{s}\right)$. This is one of the main key factors determining the operation and performance of device structure. This can be derived from solving selfconsistent Schrodinger's and Poisson's equations in the triangular potential well.

In model development, considered three quantum-well energy sub-bands say $E_{0}, E_{1}$, and $E_{2}$ to develop $n_{s}$ model 
using density-of-states function (DOS) related to FermiDirac distribution function $\mathrm{f}(\mathrm{E})$ and sub-bands and is represented as,

$$
\begin{aligned}
n_{S}=1 . \text { DOS } . \int_{E_{0}}^{E_{1}} & \frac{1}{\exp \left(\frac{E-E_{f}}{V_{T}}\right)+1} \cdot d E \\
& +2 . D O S \cdot \int_{E_{1}}^{E_{2}} \frac{1}{\exp \left(\frac{E-E_{f}}{V_{T}}\right)+1} \cdot d E \\
& +3 . \text { DOS } \cdot \int_{E_{2}}^{E_{\infty}} \frac{1}{\exp \left(\frac{E-E_{f}}{V_{T}}\right)+1} \cdot d E
\end{aligned}
$$

Where $D O S=\frac{q \cdot m_{e}^{*}}{\pi \cdot \hbar^{2}}$ and $f(E)=\frac{1}{\exp \left(\frac{E-E_{f}}{V_{T}}\right)}$

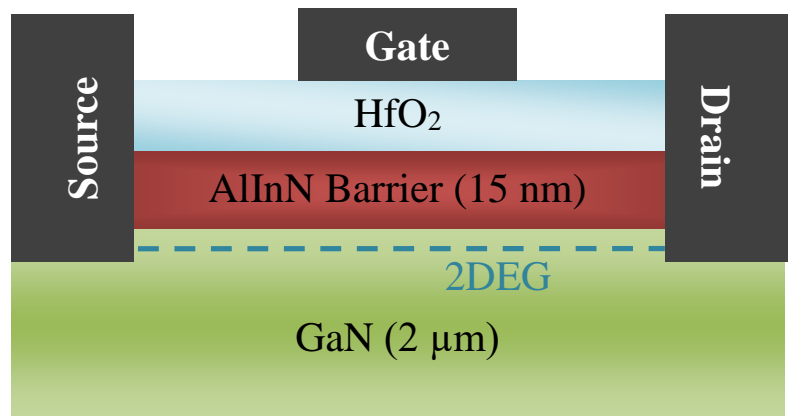

Fig. 1. Schematic view of AlInN/GaN MOSHEMT device structure.

In equation (1), neglecting the higher terms, means above $E_{2}$ state, and applying the limits, we get,

$$
\begin{aligned}
n_{S}=\operatorname{DoS} V_{T} \cdot\{ & \ln \left[\exp \left(\frac{E_{f}-E_{0}}{V_{T}}\right)+1\right] \\
& +\ln \left[\exp \left(\frac{E_{f}-E_{1}}{V_{T}}\right)+1\right] \\
& \left.+\ln \left[\exp \left(\frac{E_{f}-E_{2}}{V_{T}}\right)+1\right]\right\}
\end{aligned}
$$

Where $E_{0}=u_{0} \cdot n_{s}^{2 / 3}, E_{1}=u_{1} \cdot n_{s}^{2 / 3}$ and $E_{2}=u_{2} \cdot n_{s}^{2 / 3}$ are three lowest level energy sub-bands in the quantum-well, $E_{f}$ is fermi energy level and $V_{T}=k T / q$ thermal voltage. Assuming the AlInN layer is ionized completely, and we can express as

$$
n_{s}=\frac{\varepsilon}{q . t}\left(V_{g 0}-E_{f}\right)
$$

Where $\varepsilon=\varepsilon_{\text {Oxide }}+\varepsilon_{\text {AlInN }}, t=t_{\text {oxide }}+t_{\text {AlInN }}, \quad V_{g 0}=$ $V_{g}-V_{F B}$ and $V_{F B}$ is the flat-band voltage and assuming typical voltage of $-3.65 \mathrm{~V}[3]$.
From the equation (2) and (3), the $n_{s}$ variation with $V_{g}$ is a complicated superlative function. So, we propose an analytical model for $n_{s}$ with respect to $V_{g}$ by making simple assumptions. The $E_{f}$ and sub-band positions $E_{0}, E_{1}$ and $E_{2}$ are carried out for different $V_{g}$ are shown in figure 2. It is potent to state that the quantum-well sub-band level relative positions are in a contrast with $E_{f}$ and plotted $E_{f}-E_{0}, E_{f}-E_{1}$ and $E_{f}-E_{2}$ as shown in figure 3.

TABLE I

MODEL PARAMETERS AND THEIR DEFINITIONS

\begin{tabular}{cl}
\hline Parameter & \multicolumn{1}{c}{ Definitions } \\
\hline$n_{s}$ & 2DEG charge density $\left(\mathrm{cm}^{-2}\right)$ \\
$E_{f}$ & Fermi energy level \\
$E_{k}$ & $\mathrm{k}^{\text {th }}$ position of sub-band energy \\
$V_{T}$ & Thermal voltage, $V_{T}=k T / q$ \\
$V_{p}$ & Potential in the channel at any point $p$ \\
$W$ & Gate width, $100 \mu m$ \\
$D O S$ & Conduction band density of states, $3.24 \mathrm{e} 17$ \\
& $\mathrm{~m}^{-2} \mathrm{~V}^{-1}[12]$ \\
$\varepsilon_{\text {oxide }}$ & Permittivity of $\mathrm{HfO}_{2}, 22.3 \varepsilon_{0} F / \mathrm{m}^{2}[7]$ \\
$\varepsilon_{\text {AlInN }}$ & Permittivity of AlInN, $8.5 \varepsilon_{0} F / \mathrm{m}^{2}[8]$ \\
$t_{A l I n N}$ & Thickness of AlInN, $15 \mathrm{~nm}$ \\
$V_{F B}$ & Flat-band voltage, $-3.65 \mathrm{~V}[3]$ \\
$\mu_{0}$ & Low-field mobility, $900 \mathrm{~cm}^{2} . \mathrm{V}^{-1} \mathrm{~s}^{-1}$ \\
$A_{0}$ & Experimentally determined parameter, \\
& $2.12 e-12 \mathrm{~V} . \mathrm{m}^{4} / 3[13]$
\end{tabular}

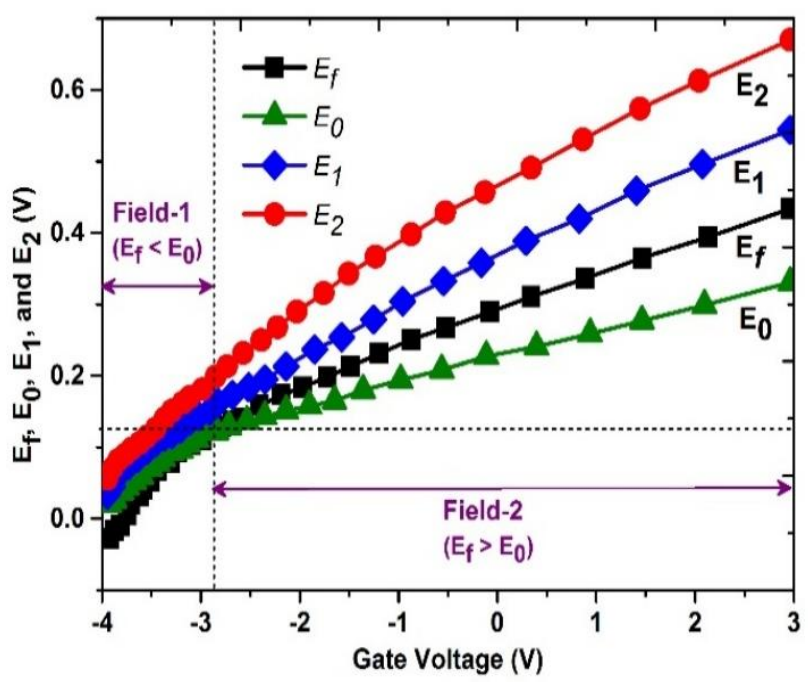

Fig. 2. $E_{f}$ and sub-band positions $E_{0}, E_{1}$, and $E_{2}$ with respect to different gate voltage. 
From figures 2 and 3, we have observed two kinds. First, the upper levels $E_{2}$ and $E_{1}$ are higher than $E_{f}$ and also with $E_{0}$ throughout the applied gate voltage. So, the contribution of higher order sub-band energy levels $E_{2}$ and $E_{1}$ are safely neglected in 2DEG charge density derivation. Second, the lowest sub-band energy level $E_{0}$ having two noticeable fields which are (i) $E_{f}<E_{0}$ and (ii) $E_{f}>E_{0}$. According to the above observations, we have developed 2DEG charge density model separately in field $1\left(E_{f}<E_{0}\right)$ and field $2\left(E_{f}>E_{0}\right)$. Then these two fields of the models are combined to develop a unified 2DEG charge density analytical model.

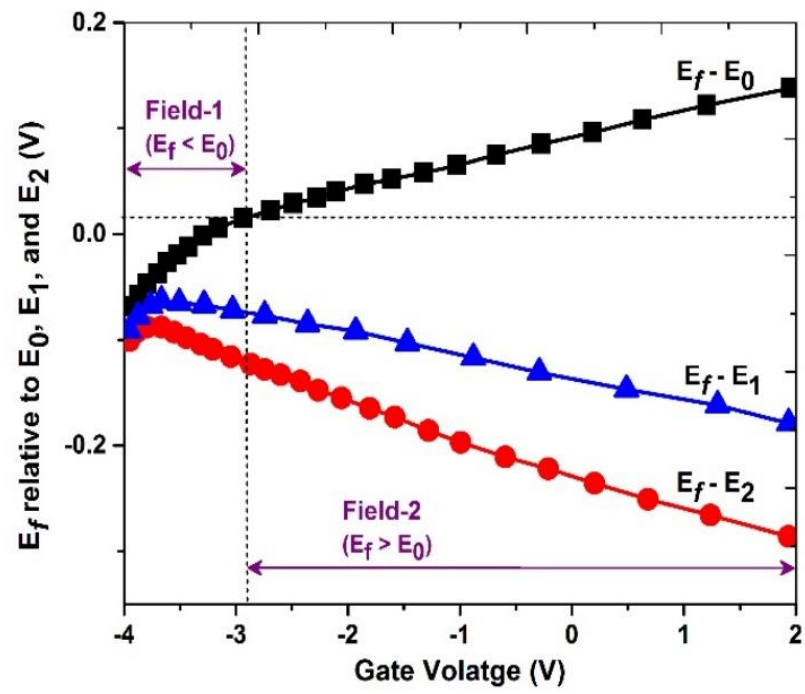

Fig. 3. Quantum-well sub-band level relative positions with $E_{f}$ of $E_{f}-E_{0}, E_{f}-E_{1}$ and $E_{f}-E_{2}$ with respect to different gate voltage.

\section{(i). Field-1 $\left(E_{f}<E_{0}\right)$ of 2DEG Charge Density Model}

From the above-mentioned observations, the contribution of higher order sub-band energy levels $E_{2}$ and $E_{1}$ are neglected. then by considering only the lowest sub band energy level term the equation (2) becomes,

$$
n_{S}=\text { DOS. } V_{T} \cdot \ln \left[\exp \left(\frac{E_{f}-E_{0}}{V_{T}}\right)+1\right]
$$

The equation (4), we can write also as

$$
\exp \left(\frac{n_{S}}{\text { DOS.V } V_{T}}\right)=\exp \left(\frac{E_{f}-E_{0}}{V_{T}}\right)+1
$$

The aim of this 2DEG charge density modelling is to develop an explicit $E_{F}$ with respect to $V_{g}$ expression as related to the precise solution as feasible, which is appropriate for drain current and other model development. Now, the Taylor's theorem approximation $\ln (1+x) \approx x$ for $x \ll 1$ is used in equation (4) and $E_{F}$ can be approximated as

$$
E_{f} \approx u_{0} \cdot n_{s}^{2 / 3}+V_{T} \cdot \ln \left(\frac{n_{s}}{\text { DOS. } V_{T}}\right)
$$

Next, the equation (3) is substituted in equation (6) to eliminate $n_{s}$ term.

$E_{f}=u_{0}\left[\frac{\varepsilon}{q . t}\left(V_{g 0}-E_{f}\right)\right]^{2 / 3}+V_{T} \cdot \ln \left[\frac{\frac{\varepsilon}{q . t}\left(V_{g 0}-E_{f}\right)}{D O S \cdot V_{T}}\right]$

Now expanding the right-hand side of the two terms to first order in the form of $E_{f} / V_{g 0}$. Then we can get,

$$
\begin{aligned}
E_{f}=u_{0}\left(\frac{\varepsilon \cdot V_{g 0}}{q \cdot t}\right)^{2 / 3}-\frac{2}{3} u_{0}\left(\frac{\varepsilon \cdot V_{g 0}}{q \cdot t}\right)^{2 / 3} \frac{E_{f}}{V_{g 0}} \\
+V_{T} \ln \left(\frac{\varepsilon \cdot V_{g 0}}{\text { q.t.DOS. } V_{T}}\right)-V_{T} \frac{E_{f}}{V_{g 0}}
\end{aligned}
$$

With further simplifications of equation (8), the $E_{f}$ can be written as

$$
E_{f}=V_{g o} \frac{V_{T} \ln \left(\frac{\varepsilon \cdot V_{g 0}}{q \cdot t \cdot D O S \cdot V_{T}}\right)+u_{0}\left(\frac{\varepsilon \cdot V_{g 0}}{q \cdot t}\right)^{2 / 3}}{V_{g 0}+\frac{2}{3} u_{0}\left(\frac{\varepsilon \cdot V_{g 0}}{q \cdot t}\right)^{2 / 3}+V_{T}}
$$

To derive the $n_{s}$ expression with respect to $V_{g}$, the equation (9) is substitute in (3) and we get,

$n_{S}=\frac{\varepsilon \cdot V_{g o}}{q . t} \cdot \frac{V_{g o}+V_{T}\left(1-\ln \left(\frac{\varepsilon \cdot V_{g 0}}{q . t . D O S . V_{T}}\right)\right)-\frac{u_{0}}{3}\left(\frac{\varepsilon \cdot V_{g 0}}{q . t}\right)^{2 / 3}}{V_{g 0}+\frac{2}{3} u_{0}\left(\frac{\varepsilon \cdot V_{g 0}}{q . t}\right)^{2 / 3}+V_{T}}$

\section{(ii). Field-2 $\left(E_{f}>E_{0}\right)$ of $2 D E G$ Charge Density Model}

In field-2, it is noticed that the $E_{f}$ is greater than $E_{0}$. By applying the first and second observations to the equation (2), the $n_{s}$ expression approximated as,

$n_{s} \approx \operatorname{DOS} .\left(E_{f}-E_{0}\right)$

To derive the $E_{f}$ and $n_{s}$ expression with respect to $V_{g}$ use the equation (11) and (3)

$$
\frac{\varepsilon}{q . t}\left(V_{g 0}-E_{f}\right)=\operatorname{DOS}\left(E_{f}-u_{0} \cdot n_{s}^{2 / 3}\right)
$$


The $E_{f}$ expression with respect to $V_{g}$ in field-2 is derived from equation (12) and the same approach is used as in field-1, and written as

$$
E_{f}=V_{g o} \frac{\frac{\varepsilon \cdot V_{g 0}}{q \cdot t \cdot D O S}+u_{0}\left(\frac{\varepsilon \cdot V_{g 0}}{q \cdot t}\right)^{2 / 3}}{\left(\frac{\varepsilon}{q \cdot t \cdot D O S}+1\right) V_{g 0}+\frac{2}{3} u_{0}\left(\frac{\varepsilon \cdot V_{g 0}}{q \cdot t}\right)^{2 / 3}}
$$

Then, the equation (13) is substitute in (3) to get field-2 of $n_{s}$ model with respect to $V_{g}$ as,

$n_{S}=\frac{\varepsilon \cdot V_{g o}}{q . t} \cdot \frac{V_{g o}-\frac{u_{0}}{3}\left(\frac{\varepsilon \cdot V_{g 0}}{q . t}\right)^{2 / 3}}{\left(\frac{\varepsilon}{q . t . D O S}+1\right) V_{g 0}+\frac{2}{3} u_{0}\left(\frac{\varepsilon \cdot V_{g 0}}{q . t}\right)^{2 / 3}}$

\section{(iii). Unified 2DEG Charge Density Model}

To improve the validity of models developed in $E_{f}<E_{0}$ and $E_{f}>E_{0}$ fields, we developed a unified $n_{s}$ model by combining these two fields of the models.

Now, combining the equations (10) and (14), we get

$n_{S}=\frac{\varepsilon \cdot V_{g o}}{q . t} \cdot \frac{V_{g o}+V_{T}\left(1-\ln \left(\frac{\varepsilon \cdot V_{g 0}^{n}}{q . t . D O S . V_{T}}\right)\right)-\frac{u_{0}}{3}\left(\frac{\varepsilon \cdot V_{g 0}}{q . t}\right)^{2 / 3}}{V_{g 0}\left(\frac{V_{T}}{V_{g 0}^{d}}+1\right)+\frac{2}{3} u_{0}\left(\frac{\varepsilon \cdot V_{g 0}}{q . t}\right)^{2 / 3}}$

The equation (15) is considered to be a unified 2DEG charge density model when $V_{g 0}^{n}=\frac{\text { e.q.t.DOS. } V_{T}}{\varepsilon}$ and $V_{g 0}^{d}=$ $\frac{\text { q.t.DOS. }}{\varepsilon}$.

\section{RESULTS AND DISCUSSIONS}

The transport is primarily by the $2 \mathrm{DEG}$ charge density in hetero-structure devices with drift and diffusion currents subject to the terminal biases. The 2DEG charge density is the key principle to the further heterostructures device study. The $n_{s}$ is model is developed separately in field 1 $\left(E_{f}<E_{0}\right)$ and field $2\left(E_{f}>E_{0}\right)$. Then these two models are combined to develop a unified 2D electron gas charge density model. The developed 2DEG charge density model results are compared with TCAD [14] simulation results to validate the models. The device structure is having a $15-\mathrm{nm}$ thickness of AlInN barrier layer throughout.

Drift-diffusion numerical model including carrier continuity equation and Poisson equation is used for the simulation of carrier transport in the channel at $300 \mathrm{~K}$. Newton method is used for model calculations. Mesh value of the structure is carefully chosen by the Deck Build Editor for the accurate simulation of the important regions of the device as well as to accelerate the computational efficiency. Simulations have been carried out by using the physical models such as, FLDMOB for the electric field-dependent mobility, CONMOB for the concentration dependent mobility, and CVT-a stand-alone model which incorporates all the effects required for simulating the carrier mobility, SRH and Auger for recombination and generation. The results are analysed in Tony plot and for output data extraction the Tony plot export feature is used. During simulation, the fixed oxide charges $\left(V_{g}\right.$ independent) of $9.8 \times 10^{12} \mathrm{~cm}^{-2}$ for $\mathrm{HfO}_{2}$ at oxide/semiconductor interfaces is considered [15]. The polarization charges of $2.73 \times 10^{13} \mathrm{~cm}^{-2}$ are calculated and imposed across AlInN interface [16].

The $n_{s}$ is calculated with respect to $V_{g}$ as per equations (10), (14) and (15). These developed model results are shown in figure 4 and these results are compared with TCAD simulation results with respect to $V_{g}$. From these results, the $n_{s}$ is increasing linearly with gate voltage and the maximum charge density is in the order of $10^{13} \mathrm{~cm}^{-2}$ which is widely suitable for fabricated $\mathrm{GaN}$ based MOSHEMT devices. The figure 4 shows the developed model results and simulation results are in good agreement.

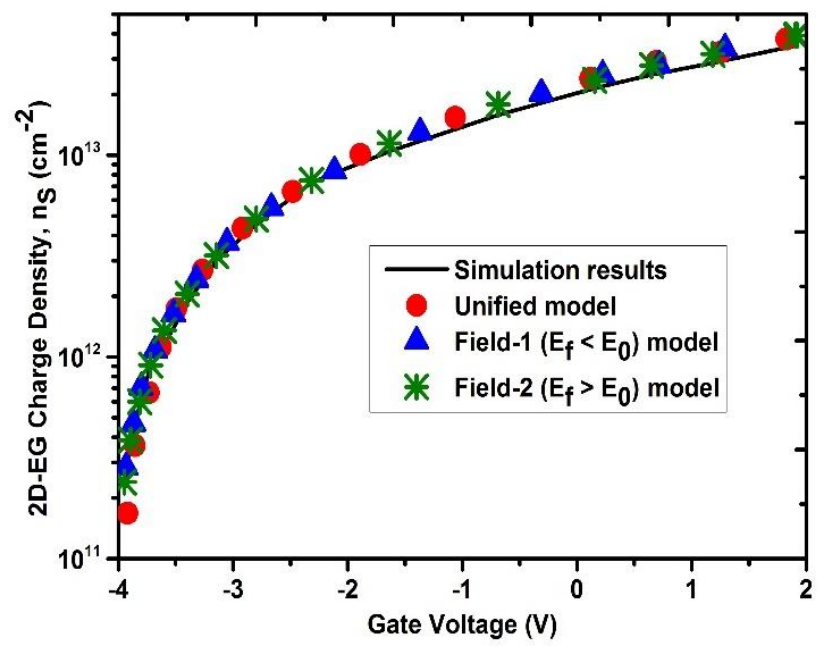

Fig. 4. Comparison of simulation and model results of $n_{s}$ with respect to $V_{g}$.

The developed models with respect to the present context the 2DEG charge density depend on thickness of the material and type of material which decides the 
dielectric permittivity and interface charges. we consider AlInN barrier material of $15 \mathrm{~nm}$ thickness and permittivity $\varepsilon_{\text {AlInN }}=8.5 \varepsilon_{0} \mathrm{~F} / \mathrm{m}^{2}$. The $\mathrm{HfO}_{2}$ material is used as oxide layer having permittivity of $\varepsilon_{\text {Oxide }}=22.3 \varepsilon_{0} F /$ $m^{2}$. In view of this, we have varied the oxide thickness from 0 to $20 \mathrm{~nm}$ with respect to $n_{s}$ and shown in figure 5 .

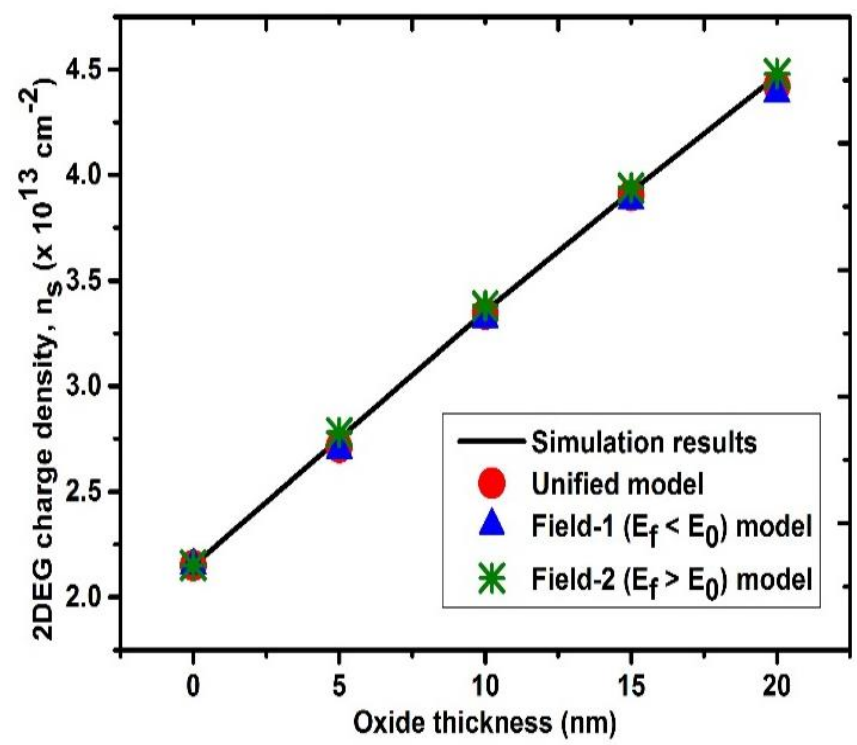

Fig.5. $n_{s}$ with respect to different oxide thicknesses.

The $n_{s}$ increasing linearly with positive slope when increasing the oxide thickness. When the oxide thickness is increasing, the electric field decreases due to the existence of positive interface and bulk oxide charges in $\mathrm{HfO}_{2}$ material [7]. When the electric field decreases the quantum-well sub-band energy values decreases then the $E_{f}$ difference increases and in consequence to increase in $n_{s}$. From figure 5 , the $n_{s}$ with respect to oxide thickness variation in two different fields and unified model results are compared with simulation results and are in good agreement. From all these results, it is important to note that the developed models can be useful to study the transport characteristics of III-Nitride based MOSHEMT structures.

\section{CONCLUSION}

We have developed an analytical model for the unified 2D electron gas charge density of $\mathrm{HfO}_{2} / \mathrm{AlInN} / \mathrm{GaN}$ MOSHEMT device. The unified $n_{s}$ model is developed by solving transcendental relation of $n_{s}$ and $E_{f}$ with respect to gate voltage in field $1\left(E_{f}<E_{0}\right)$ and field $2\left(E_{f}>E_{0}\right)$ and combined them. This model has not required any fitting or empirical parameters. These developed models are verified with different oxide thickness and shows a linear positive increment in $n_{s}$ with increasing the oxide thickness. The developed model results are compared with simulation results and found in a good agreement between them. Finally, it is important to note that the developed models can be useful to study the further characteristics of III-N based MOSHEMT structures.

\section{ACKNOWLEDGEMENT}

The authors acknowledge the Microelectronics Computational Laboratory of Department of ECE, National Institute of Technology, Silchar, India by providing necessary facilities to carry out the research work.

\section{REFERENCES}

[1]. K. Jena, R. Swain, and T. R. Lenka, "Physics-based mathematical model of 2DEG sheet charge density and DC characteristics of AlInN/AlN/GaN MOSHEMT: Sheet Charge Density and DC Characteristics of AlInN/AlN/GaN MOSHEMT," International Journal of Numerical Modelling: Electronic Networks, Devices and Fields, vol. 30, no. 1, p. e2117, Jan. 2017.

[2]. Y. Yue et al., "InAlN/AIN/GaN HEMTs With Regrown Ohmic Contacts and fT of $370 \mathrm{GHz}$," IEEE Electron Device Letters, vol. 33, no. 7, pp. 988-990, Jul. 2012.

[3]. H. Zhou et al., "High-Performance InAlN/GaN MOSHEMTs Enabled by Atomic Layer Epitaxy $\mathrm{MgCaO}$ as Gate Dielectric," IEEE Electron Device Letters, vol. 37, no. 5, pp. 556-559, May 2016.

[4]. S. Yagi et al., "High breakdown voltage AlGaN/GaN MISHEMT with $\mathrm{SiN}$ and $\mathrm{TiO} 2$ gate insulator," Solid-State Electronics, vol. 50, no. 6, pp. 1057-1061, Jun. 2006.

[5]. F. Husna, M. Lachab, M. Sultana, V. Adivarahan, Q. Fareed, and A. Khan, "High-Temperature Performance of AlGaN/GaN MOSHEMT With $\mathrm{SiO} 2$ Gate Insulator Fabricated on Si (111) Substrate," IEEE Transactions on Electron Devices, vol. 59, no. 9, pp. 2424-2429, Sep. 2012.

[6]. Y. Yue et al., "Ultrascaled InAlN/GaN High Electron Mobility Transistors with Cutoff Frequency of $400 \mathrm{GHz}$," Japanese Journal of Applied Physics, vol. 52, no. 8S, p. 08JN14, Aug. 2013

[7]. R. Swain, J. Panda, K. Jena, and T. R. Lenka, "Modeling and simulation of oxide dependent 2DEG sheet charge density in AlGaN/GaN MOSHEMT," Journal of Computational Electronics, vol. 14, no. 3, pp. 754-761, Sep. 2015.

[8]. Q. Feng, Q. Li, T. Xing, Q. Wang, J.-C. Zhang, and Y. Hao, "Performance of La2O3/InAlN/GaN metal—oxide- 
semiconductor high electron mobility transistors," Chinese Physics B, vol. 21, no. 6, p. 67305, Jun. 2012.

[9]. X.-Y. Liu et al., "AlGaN/GaN MISHEMTs with AlN gate dielectric grown by thermal ALD technique," Nanoscale Research Letters, vol. 10, no. 1, Dec. 2015.

[10]. Shu Yang et al., "AlGaN/GaN MISHEMTs With High-k LaLuO3 Gate Dielectric," IEEE Electron Device Letters, vol. 33, no. 7, pp. 979-981, Jul. 2012.

[11]. J. Zhang, B. Syamal, X. Zhou, S. Arulkumaran, and G. I. $\mathrm{Ng}$, "A Compact Model for Generic MIS-HEMTs Based on the Unified 2DEG Density Expression," IEEE Transactions on Electron Devices, vol. 61, no. 2, pp. 314-323, Feb. 2014.

[12]. W. Deng, J. Huang, X. Ma, J. J. Liou, and F. Yu, “A compact drain current model for heterostructure HEMTs including 2DEG density solution with two subbands," Solid-State Electronics, vol. 115, pp. 54-59, Jan. 2016.
[13].S. Khandelwal and T. A. Fjeldly, "A physics based compact model of $\mathrm{I}-\mathrm{V}$ and $\mathrm{C}-\mathrm{V}$ characteristics in AlGaN/GaN HEMT devices," Solid-State Electronics, vol. 76, pp. 60-66, Oct. 2012.

[14]. User Guide Manual, ATLAS, Version 5.12.0.R./USA, Silvaco Inc, 2010.

[15].M. Tapajna, J. Kuzmík, “A Comprehensive Analytical Model for Threshold Voltage Calculation in GaN Based Metal-Oxide-Semiconductor High-Electron-Mobility Transistors," Applied Physics Letters, vol. 100, pp. 1135091-4, 2012.

[16]. J. Wang et al., "Evidence of Type-II Band Alignment in III-nitride Semiconductors: Experimental and theoretical investigation for In $0.17 \mathrm{Al} 0.83 \mathrm{~N} / \mathrm{GaN}$ heterostructures," Scientific Reports, vol. 4, p. 6521, Oct. 2014. 